\title{
Brightening and controlling dark excitons in monolayer TMDCs
}

\author{
Qing-Hai $\operatorname{Tan}^{1,2}$ and Jun Zhang ${ }^{1,2^{*}}$
}

An exciton is a bound state formed by an excited electron and hole via the Coulomb attraction. Such bound states often play an important role in the optical properties of low dimensional materials such as quantum dots, nanowires and two-dimensional (2D) materials, owing to their strong spatial confinement and reduced screening effect compared to bulk sample. The 2D nature of transition metal dichalcogenides (TMDCs) brings a tightly bound exciton with large exciton binding energy. At the simplest level, if an electron-hole pair interacts through a Coulomb attractive central potential, it will form a series of excitonic Rydberg-like states with definite parity, similar to the hydrogen model [1]. Usually we can observe A, B and $\mathrm{C}$ excitons in those TMDCs materials. $\mathrm{A}$ and $\mathrm{B}$ features involve the band edge states at $K$ points, while the $\mathrm{C}$ exciton is related to the parallel bands near $\Gamma$ point at higher energies. According to the selection rule of dipole transition, these exciton states either are optical transition allowed bright excitons or optical transition forbidden dark excitons depending on the relative spin orientation of electrons in conduction and valence band [2-4]. In short, bright excitons correspond to the transition from electron- hole pairs with the same spin orientation, while the dark exciton corresponds to the transition from electron-hole pairs with the opposite spin orientation. Generally, the dark excitons have a much longer lifetime, which makes it to have potential applications in quantum information processing [5]. Meanwhile, strong spin-orbit coupling in monolayer TMDCs leads to a larger spin splitting of the valance band and a much smaller splitting of the lowest conduction band at $K$ point [6]. In $\mathrm{WX}_{2}$ $(\mathrm{X}=\mathrm{S}, \mathrm{Se})$, since electrons in the lowest conduction band are expected to have the opposite spin to holes in the highest valance band, it forms dark A exciton $\left(\mathrm{X}_{\mathrm{D}}\right)$ that is out-plane ( $\mathrm{Z}$ direction) polarized and optically forbidden for the in-plane (X-Y plane) polarized excitation [2,3]. In $\mathrm{MoX}_{2}(\mathrm{X}=\mathrm{S}, \mathrm{Se})$, the spin orientations of this two states are parallel each other and form bright $\mathrm{A}$ exciton $\left(\mathrm{X}_{0}, \mathrm{X}-\mathrm{Y}\right.$ plane polarization), but the dark state lies at the higher energy level of split conduction band, as shown in Fig. 1a.

The $\mathrm{Z}$ polarized dark excitons are difficult to be directly observed with normal incident excitation. However, we should note that dark $\mathrm{A}$ exciton $\mathrm{X}_{\mathrm{D}}$ slightly mixes different orbital and spin wave functions due to the spin orbit coupling. As a result, the oscillator strength of the corresponding transition is quite small but not completely forbidden transition [3]. Therefore, there are still some possibilities to directly observe the dark A exciton $\mathrm{X}_{\mathrm{D}}$ under certain experimental configuration. Actually, several groups have independently observed the $X_{D}$ with different experimental methods. Both of Heinz's group and Molas's group applied a large in-plane magnetic field (>14 $\mathrm{T})$ to induce the mixing oscillator strength of bright and dark state pairs and lead to $\mathrm{X}_{\mathrm{D}}$ in $\mathrm{WX}_{2}$ with a weak in-plane polarization and become bright $[7,8]$. Similar technique has already been successfully applied to observe the dark exciton in quantum dots [9]. Meanwhile, Kim and co-workers probed dark excitons in atomically thin semiconductors via near-field coupling to surface plasmon polaritons (SPP) by placing a monolayer $\mathrm{WSe}_{2}$ on the atomically smooth single-crystal silver film [10]. Urbaszek et al. [11,12] observed $\mathrm{X}_{\mathrm{D}}$ emission in $\mathrm{WX}_{2}$ by using the polarization-selective detection from the sample edge. However, owing to the small splitting of conduction band and the thermal excitation effects, all of these experiments are limited within low temperature $(<30 \mathrm{~K})$.

Recently, Raschke and his colleagues [13] from University of Colorado, Boulder in USA reported the observation of dark exciton in monolayer $\mathrm{WSe}_{2}$ at room temperature by combining the near-field optics and

\footnotetext{
${ }^{1}$ State Key Laboratory of Superlattices and Microstructures, Institute of Semiconductors, Chinese Academy of Sciences, Beijing 100083, China

${ }^{2}$ CAS Center of Excellence in Topological Quantum Computation, College of Materials Science and Opto-Electronic Technology, University of Chinese Academy of Sciences, Beijing 101408, China

*Email: zhangjwill@semi.ac.cn
} 
a

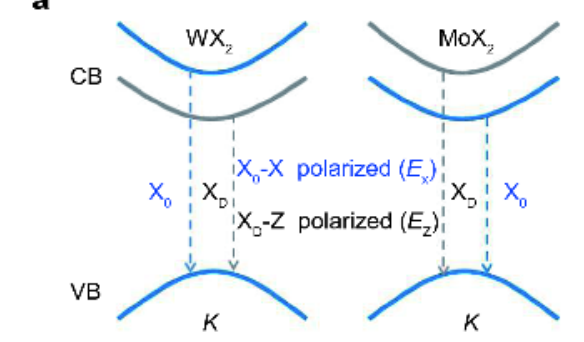

b

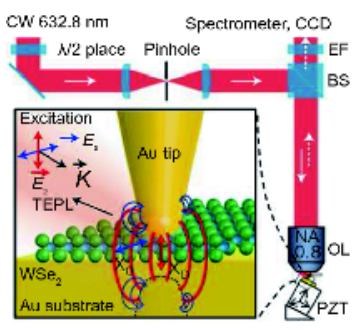

Wavelength $(\mathrm{nm})$ C 850800750700

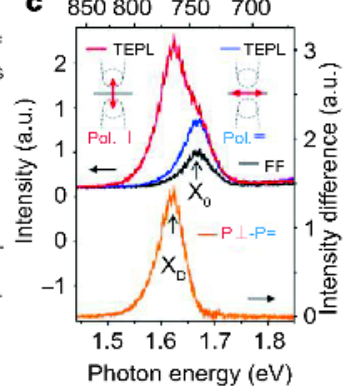

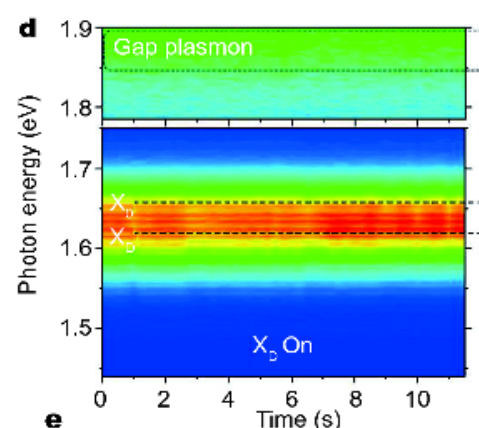
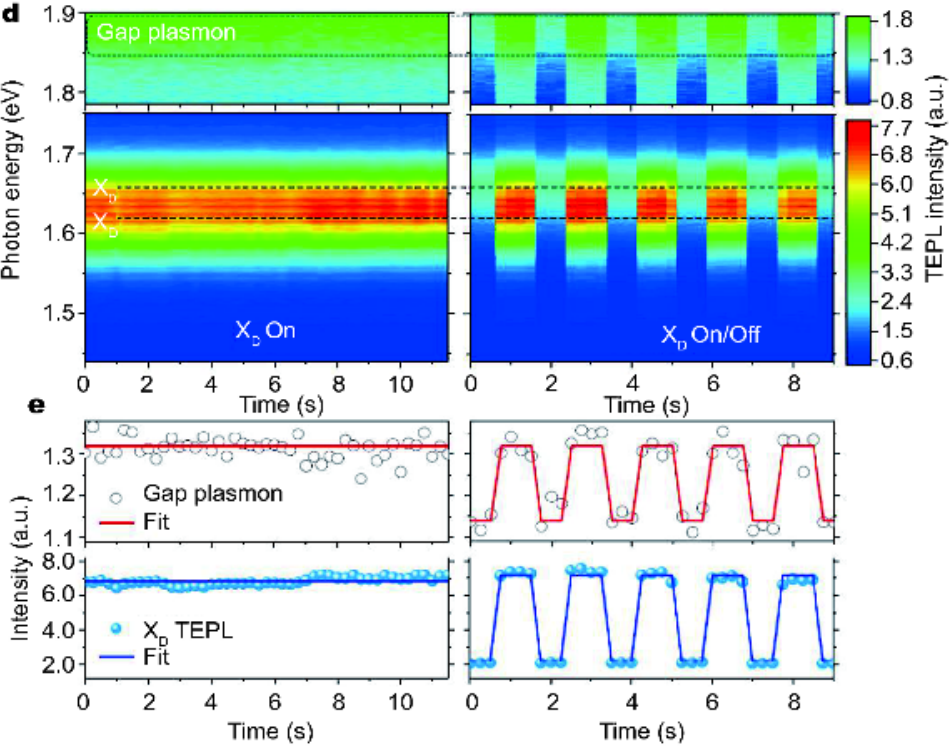

Figure 1 (a) The splitting of conduction band at around $K$ point and the transition of bright $\left(\mathrm{X}_{0}\right)$ and dark $\left(\mathrm{X}_{\mathrm{D}}\right)$ exciton in $\mathrm{WX} \mathrm{X}_{2}$ and $\mathrm{MoX}_{2}$, respectively. (b) Schematic of tip-enhanced photoluminescence (TEPL) spectroscopy, selective excitation and probing of the transition dipole moments of dark (out-of-plane) and bright (in-plane) excitons by polarization control. (c) Excitation polarization dependent TEPL spectra of monolayer $\mathrm{WSe}_{2}$ on an Au substrate at $\sim 1 \mathrm{~nm}$ tip-sample distance with tip selective $\mathrm{X}_{\mathrm{D}}$ emission (orange). (d) Time-series TEPL response $\left(\mathrm{X}_{\mathrm{D}}\right.$, $\mathrm{X}_{0}$ and gap plasmon $\mathrm{PL}$ ) in continuous $\mathrm{On}$ state of $\mathrm{X}_{\mathrm{D}}$ emission at $1 \mathrm{~nm}$ tip-sample distance and discrete On/Off switching of $\mathrm{X}_{\mathrm{D}}$ emission with discrete tipsample distance variation between $1 \mathrm{~nm}$ and $5 \mathrm{~nm}$, respectively. (e) Time-series of peak intensity of TEPL response with correlation between corresponding $\mathrm{X}_{\mathrm{D}}$ emission and gap plasmon, derived from d. Reprinted with permission from Ref. [13], Copyright 2017, Nature Publishing Group.

changing the polarization of excitation light relative to the sample surfaces. Fig. $1 \mathrm{~b}$ shows their schematic of tipenhanced photoluminescence (TEPL) spectroscopy, where the polarization of excitation light is changed by $\lambda / 2$ plate. The samples are mounted to a piezoelectric transducer (PZT) and the sample surface is tilted by $\sim 60^{\circ}$ with respect to the incident $\boldsymbol{k}$-vector for effective excitation. The distance between tip and sample was controlled electrically with $\sim 0.2 \mathrm{~nm}$ precision. When the distance is fixed at around $1 \mathrm{~nm}$, the dark exciton can couple to laser with $\mathrm{Z}$ polarization component at room temperature, as shown in Fig. 1c. In this case, the transition dipole moment and the spontaneous emission rate of dark exciton are greatly enhanced by this nano-gap due to the plasmonic Purcell effect. In particular, the nanocavity bellow $1.5 \mathrm{~nm}$ gap gives rise to a large Purcell factor $(>2,000)$ compared to dielectric substrates and lead to a huge enhancement of the dark excitons emission. Remarkably, by switching the tip distance between $1 \mathrm{~nm}$ and $5 \mathrm{~nm}$, they have achieved the switching between On and Off state of $\mathrm{X}_{\mathrm{D}}$, as shown in Fig. 1d, e. Furthermore, the intensity of $\mathrm{X}_{\mathrm{D}}$ emission can be programmably controlled by changing this gap distance. This work provides a new way to actively control the emission of dark excitons by changing the polarization of laser and the distance of between plasmonic tip and sample surfaces, which may broaden potential applications of dark excitons in information processing. As pointed by the authors, similar method of brightening and controlling the dark excitons could be applied to other semiconductors, such as carbon nanostructures, quantum dots, other van der Waals materials beyond TMDCs.

In addition to the direct measurement of dark exciton, Zhang and colleges [14] also indirectly measured the $X_{D}$ and related energy splitting between dark and bright $\mathrm{A}$ exciton in few-layer $\mathrm{WS}_{2}$ by using resonant Raman scattering technique, where the selection rule of dipole transiton is satisfied by coupling odd-parity infrared active phonons and even parity dark excitons. From above reports, it must also be noted that the $\mathrm{X}_{\mathrm{D}}$ in $\mathrm{MoX}_{2}$ is still hard to be observed in experiment, because the dark states in $\mathrm{MoX}_{2}$ lies at the higher energy compared to bright states, which leads to a lower population and thus lower transition probability $[2,3,12]$. In order to brighten and control these $\mathrm{X}_{\mathrm{D}}$ in $\mathrm{MoX}_{2}$, a feasible way is to combine exciton-phonons coupling with special experimental 
configuration such as tip-enhanced exciton-SPP coupling strain field. In future, an important direction is to develop more easy and reliable techniques to brighten and control the dark exciton in $2 \mathrm{D}$ and $2 \mathrm{D}$ van der Waals heterostructures.

Received 4 April 2018; accepted 20 April 2018; published online 11 May 2018

1 Ye Z, Cao T, O'Brien K, et al. Probing excitonic dark states in single-layer tungsten disulphide. Nature, 2014, 513: 214-218

2 Dery H, Song Y. Polarization analysis of excitons in monolayer and bilayer transition-metal dichalcogenides. Phys Rev B, 2015, 92: 125431

3 Echeverry JP, Urbaszek B, Amand T, et al. Splitting between bright and dark excitons in transition metal dichalcogenide monolayers. Phys Rev B, 2016, 93: 121107

4 Nirmal M, Norris DJ, Kuno M, et al. Observation of the "dark exciton" in CdSe quantum dots. Phys Rev Lett, 1995, 75: 37283731

5 Heindel T, Thoma A, Schwartz I, et al. Accessing the dark exciton spin in deterministic quantum-dot microlenses. APL Photonics, 2017, 2: 121303

6 Mak KF, Lee C, Hone J, et al. Atomically thin $\mathrm{MoS}_{2}$ : A new direct- gap semiconductor. Phys Rev Lett, 2010, 105: 136805

7 Molas MR, Faugeras C, Slobodeniuk AO, et al. Brightening of dark excitons in monolayers of semiconducting transition metal dichalcogenides. 2D Mater, 2017, 4: 021003

8 Zhang XX, Cao T, Lu Z, et al. Magnetic brightening and control of dark excitons in monolayer $\mathrm{WSe}_{2}$. Nat Nanotechnol, 2017, 12: 883888

9 Peng K, Wu S, Tang J, et al. Probing the dark-exciton states of a single quantum dot using photocurrent spectroscopy in a magnetic field. Phys Rev Appl, 2017, 8: 064018

10 Zhou Y, Scuri G, Wild DS, et al. Probing dark excitons in atomically thin semiconductors via near-field coupling to surface plasmon polaritons. Nat Nanotechnol, 2017, 12: 856-860

11 Robert C, Amand T, Cadiz F, et al. Fine structure and lifetime of dark excitons in transition metal dichalcogenide monolayers. Phys Rev B, 2017, 96: 155423

12 Wang G, Robert C, Glazov MM, et al. In-plane propagation of light in transition metal dichalcogenide monolayers: optical selection rules. Phys Rev Lett, 2017, 119: 047401

13 Park KD, Jiang T, Clark G, et al. Radiative control of dark excitons at room temperature by nano-optical antenna-tip Purcell effect. Nat Nanotechnol, 2018, 13: 59-64

14 Tan QH, Sun YJ, Liu XL, et al. Observation of forbidden phonons, Fano resonance and dark excitons by resonance Raman scattering in few-layer $\mathrm{WS}_{2}$. 2D Mater, 2017, 4: 031007 